\title{
Psychological factors associated with malignant fungating breast wounds
}

Keywords: fungating wounds, psychosocial effects, body image, femininity

Malignant fungating wounds (MFWs) can be a distressing consequence of breast cancer. Although the true prevalence of MFWs is unknown, existing figures suggest that rates may be as high as $62 \%$, and this figure increases with age. Multiple studies have examined the psychological consequences of MFWs in the breast. This review presents a summary of some of the research to examine the role of specialist wound advice.

\begin{abstract}
\section{Background}

Malignant fungating breast wounds, or malignant fungating wounds (MFWs) hereinafter, indicate a poor prognosis. For patients, malodour and copious exudate are the common distressing symptoms that require careful management. In addition to local wound management, patients require sensitive psychological support to improve their quality of life.
\end{abstract}

\section{Aim}

To review the current evidence on the psychological effects of MFWs.

\section{Findings}

In women with an MFW, feelings of anxiety associated with malodour and exudate were common, which affected their femininity and restricted their choice of clothing. In turn, this affected their body image and identity, in addition to creating a sense of social isolation, shame, and embarrassment. Moreover, the presence of an MFW served as a constant reminder of impending death, and the risk of bleeding was a major concern. Multiple studies have identified the positive influences of specialist and supportive care.

\section{Conclusion}

The evidence suggests that the main psychological aspects of an MFW in the breast are associated with the symptoms and visual nature of the wound, which act as a perpetual reminder of the person's disease and mortality. The studies reviewed herein demonstrate how an MFW affects a woman's femininity, body image, and self-esteem, leading to feelings of embarrassment, fear, and shame, and consequently, social isolation. The implications for clinical practice that emerged from the review highlight the importance of the management of wound malodour and exudate and also draw attention to the importance of access to specialist wound care support as these have been shown to have a positive effect on quality of life and mitigate some of the psychosocial effects of MFW.

\section{Key messages}

This review explored the psychological factors associated with an MFW of the breast. The results highlight that individuals with MFW experience low self-esteem the effects of which extend into many aspects of their daily lives, including their sex lives and social interactions. For many patients odour and exudate were the main contributing factors to feelings of shame and embarrassment, Furthermore for those individuals who experienced bleeding as a symptom of their wound reported being frightened of bleeding to death. A number of studies were able to show the positive effects of specialist wound care and counselling on the psychological wellbeing of the affected women.

\section{INTRODUCTION}

In the UK, 54,751 cases of breast cancer in the female population were reported in $2015 .^{1}$ The incidence rates of breast cancer increase progressively from the ages of 30-34 years and more remarkably from the ages of $70-74$ years. ${ }^{1}$ During $2016,11,563$ deaths occurred in relation to breast cancer, accounting for $7 \%$ of all cancer-related deaths, with mortality peaking at 90 plus years of age. ${ }^{1}$

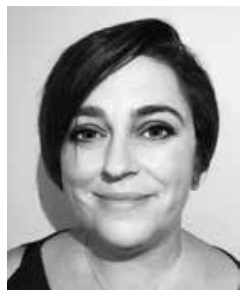

Pauline J Robinson RN, Year 1 Student Masters in Wound Healing and Tissue Repair Centre for Medical Education, School of Medicine, Cardiff University Wales, UK

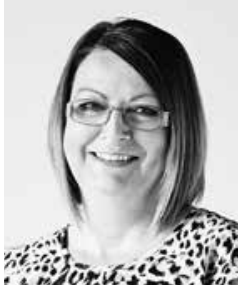

Samantha L Holloway MSc, Reader, Programme Director. Masters in Wound Healing and Tissue Repair Centre for Medical Education, School of Medicine Cardiff University, Wales, UK

Correspondence: hollowaysl1@cf.ac.uk

Conflicts of interest: None 
Malignant fungating wounds (MFW) are a distressing complication of cancer. and have been mostly commonly reported in individuals with breast cancer. ${ }^{2}$ The prevalence of MFWs in breast cancer patients has been reported to be between $47.1 \%^{3}$ to $62 \%{ }^{4}$ The paucity of literature on the psychological effects of MFW is evident as is the lack of knowledge pertaining to the management of these wounds among healthcare professionals. ${ }^{4}$

MFW are caused by the infiltration of malignant cells into the skin and adjacent blood and lymph vessels, and they are normally an indicator of a poor prognosis. ${ }^{5-7}$ Malignant MFW can occur as a consequence of advanced primary, secondary, or recurrent cancer, and treatment is mainly palliative. ${ }^{6,7}$ Unless the infiltration of cells is managed with adjuvant treatments, such as chemotherapy, radiotherapy, hormone therapy, or surgery, an MFW will continue to grow, causing further damage to the surrounding tissues. ${ }^{6}$

MFW can have wide-ranging effects on the physical and emotional wellbeing of the affected person, as well as those of the family members and healthcare professionals. ${ }^{7}$ Such wounds are associated with distressing symptoms such as odour, exudate, pain, bleeding, and itching, and they affect a person's body image and self-esteem, often leading to depression and social isolation. ${ }^{6}$ According to a number of authors, knowledge and consideration among healthcare professionals of these often distressing physical and psychological symptoms will help improve the quality of life of this patient group. ${ }^{6,7}$ This review explores the psychological factors associated with an MFW of the breast.

\section{PSYCHOSOCIAL EFFECTS OF MALIGNANT FUNGATING BREAST WOUNDS}

Lund-Neilsen et al. undertook a prospective, exploratory intervention study to investigate the effects of MFW on femininity, sexuality, and daily life in 12 women with advanced breast cancer. ${ }^{8}$ Over a 4 -week period, the women received psychological support in addition to evidencebased wound care. The authors conducted semi-structured interviews pre- and post the intervention period to enhance data capture. The responses indicated that the women expressed feelings of anxiety associated with malodour and exudate. Moreover, the women described how this affected their femininity and restricted their clothing choices, which affected their identity and created a sense of social isolation. Participants over the age of 70 years felt these were not major issues, but they acknowledged that if they were younger, the effects would be considerably stronger. With regards to sexuality, four of the women had lost their partners and lived alone. One-third of the women felt the MFW had a negative effect on their sex life, with the adverse symptoms of the MFW such as malodour and exudate being the main contributing factors.
Because of these factors, it was impossible for the women to be intimate with their partners, and their sexual desires faded. The study drew attention to the low self-esteem of the affected women and the effects of MFWs on their daily lives, including their sex lives and social interactions. In addition, the authors discussed the positive effects of specialist wound care and counselling on the psychological wellbeing of the affected women. The interventions were conducted in the women's own houses, which the authors suggested probably had a positive effect because women were more likely to reveal their inner fears and anxieties in such a safe and stress-free setting.

In a later qualitative study, Lo et al. investigated the experience of patients living with an MFW of the breast. ${ }^{4}$ Through semi-structured in-depth interviews, the authors identified a trajectory of five themes, namely, declining physical wellbeing, wound-related stigma, need for expert help, wound management strategies, and living positively with the wound. These themes were affected considerably by the wound care the participants received. The study participants described feeling socially isolated and ashamed, with odour and exudate being the main contributing factors. This, in turn, affected their confidence and body image. One participant mentioned keeping the wound a secret, another felt the wound influenced how others perceived them, and yet another felt the wound was a perpetual reminder of their diagnosis and impending death. A few participants attempted to self-manage their wounds to reduce the embarrassment and social stigma before seeking specialist wound management.

Lo et al. reported that once a specialist assessed and advised the participants on the management of their MFW, their lived experiences were influenced positively, with enhanced emotional wellbeing, improved quality of life, and increased self-confidence. This helped them to live more positively with their MFW and become less socially isolated. The importance of specialist and supportive care has also been identified in a more recent study. ${ }^{9}$

In a prospective case series, Maida et al. collected data based on patients self-reporting of the symptoms associated with MFW $(\mathrm{n}=67) .{ }^{3}$ Of these patients, $19.4 \%(\mathrm{n}=$ 13) highlighted the distress caused by the aesthetics of their wounds, especially if the dressings emphasised asymmetry. In addition, the authors reported that the patients felt embarrassed by the wound odour, leading to social isolation. These findings substantiated the findings of existing studies in terms of the psychological effects of MFW on the patients' sense of femininity and social interaction. ${ }^{4,8}$ An additional observation was that patients who experienced bleeding as a symptom of their wound reported being frightened of bleeding to death. 
In a phenomenological study, Probst et al. explored the lived experiences of nine patients with an MFW of the breast. ${ }^{10}$ The authors discussed how living with a visible cancer affects a person's quality of life and emotional wellbeing, as well as the psychological distress it causes. Probst et al. proposed that women with advanced breast cancer who delayed seeking medical care did so because of feelings of blame and shame, ignoring the MFW developing in their breast until the symptoms became insurmountable. The findings of Probst et al. substantiated those of Lo et al., who identified that odour and exudate were the main contributing factors to feelings of shame and embarrassment, as well as the fear of looming death and effect on body image. Moreover, participants in the study of Probst et al. described being averse to taking regular prescribed analgesia owing to a fear of addiction to opioids, validating the findings of a previous case series. ${ }^{3}$

The themes of living with an MFW and feeling different emerged in the study by Probst et al.. ${ }^{10}$ The authors reported how living with an MFW transformed the lives of the study participants entirely, with the graphic nature of the wound causing tremendous suffering. Some of the women found managing the distressing symptoms to be all-consuming endeavour, with one participant washing her wound every hour to eliminate the malodour and another doing the same to manage the exudate. Other participants coped by researching complementary and alternative therapies to manage the symptoms of their MFW, helping regain control of their bodies. This increased their confidence and helped them regain a sense of normality. Regaining a sense of normality was identified as a key coping mechanism, even though the women reported feel- ing different. ${ }^{10}$ Similar themes were also identified in an earlier study. ${ }^{11}$

All the women in the study by Probst et al. mentioned that they coped by keeping their wound a secret, some even from family members. Others coped through denial and the hope of restoration of health, and they found the acceptance of a poor prognosis incomprehensible. ${ }^{10}$ The authors suggested that active coping strategies such as the ones mentioned above should be encouraged by healthcare professionals, especially with regards to complementary and alternative therapies, as well as good communication skills, to tackle the psychological effects of an MFW. ${ }^{10}$

Overall, the results of Probst et al. support the results of earlier studies. ${ }^{3-4,8,11} \mathrm{~A}$ number of the studies advocated the importance of specialist wound management $t^{4,8}$, and one study mentioned good communication skills. ${ }^{10} \mathrm{How}-$ ever, no study has offered recommendations on how to support women in mitigating the psychosocial effects of their MFWs. Subsequently this was addressed in a systematic review of the available literature. ${ }^{12}$ The authors examined 10 primary studies focusing on the quality of life of adults and proposed several recommendations (Table 1).

In addition, Gibson and Green suggested the introduction of a reporting system for MFW to determine their prevalence because according to them, an understanding of the extent of the problem may help stimulate further research and the development of a national guidance. ${ }^{12}$ However, to date, the recommendation to create a registry for MFWs has seemingly not been implemented.

Table 1.

Recommendations for supporting women with MFW'12

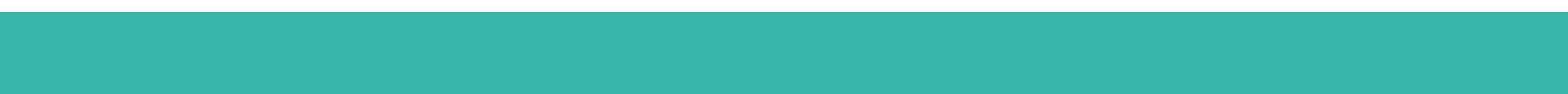

Provision of information to patients, both verbal and written

Signposting to supportive services to reduce anxiety and stress

Offering patients a simple and specific quality-of-life assessment tool to help stimulate expression of any anxieties to enable a problem-solving approach

Training and education programmes for nurses

Allowing time for care

Continual assessment of pain

Supplying an 'emergency box' with suitable dressings, medications, and dark-coloured towels in case of haemorrhage 


\section{CONCLUSION}

Overall, the evidence reviewed indicates that the majority of the psychological aspects of an MFW of the breast are associated with the symptoms and visual nature of the wound, which acts as a perpetual reminder of the person's disease and mortality (Lund-Neilson 2005, Lo et al. 2008, Maida 2009, Probst et al. 2013). However, other authors have described the anxiety among patients with young children and their fear of the effects of the disease on other people ${ }^{11}$ The literature describes how MFWs affect women's femininity, body image, and self-esteem, leaving them with feelings of embarrassment, fear, and shame, often leading to social isolation. Malodour and exudate seem to have the most profound effects. This highlights the importance of specialist wound care support and advice, which appeared to have positive effects on women's quality of life and mitigated some of the psychosocial effects of MFWs.

\section{IMPLICATIONS FOR CLINICAL PRACTICE}

Healthcare professionals need to be acutely aware of the low self-esteem that individuals with MFW experience.

Specialist and supportive care is fundamental for an individual's well-being

\section{Allowing time to care is of paramount importance}

Healthcare professionals need to acknowledge the real fear individuals have of leakage, odour and bleeding and provide solutions to these such as an 'emergency care box'.

\section{FURTHER RESEARCH}

Identification of an appropriate quality of life assessment tool for individuals with MFW to facilitate identification of patient -focussed outcome measures

Working with individuals with MFW to co-produce information leaflets suitable for both patients and family

\section{REFERENCES}

1. Cancer Research. 2018. [Accessed: 2nd January 2018]. Available at: https://www.cancerresearchuk. org/health-professional/cancer-statistics/statistics-bycancer-type/breast-cancer/mortality.

2. Thomas, S. Current Practices in the Management of Fungating Lesions and Radiotherapy Damaged Skin The Surgical Materials Testing Laboratory, Bridgend 1992

3. Maida V, Ennis M, Kuziemsky C, Trozzolo L. Symptoms associated with malignant wounds: a prospective case series. J Pain Symptom Manage. 2009 Feb 1;37(2):206-11.

4. Lo SF, Hu WY, Hayter M, Chang SC, Hsu MY, Wu LY. Experiences of living with a malignant fungating wound: a qualitative study. Journal of Clinical Nursing. 2008 Oct; 17(20):2699-2708.
5. Alexander S. Malignant fungating wounds: epidemiology, aetiology, presentation and assessment. Journal of Wound Care. 2009 Jul; 18(7):273-80.

6. Merz T, Klein C, Uebach B, Kern M, Ostgathe C Bükki J. Fungating wounds-multidimensional challenge in palliative care. Breast Care. 2011;6(1):21-4

7. Probst S, Grocott P, Graham T, Gethin G. Recommendations for the care of patients with malignant fungating wounds. European Oncology Nursing Society (EONS). 2015.

8. Lund-Nielsen B, Müller K, Adamsen L. Malignant wounds in women with breast cancer: feminine and sexual perspectives. Journal of Clinical Nursing. 2005 Jan; 14(1):56-64.

9. Reyonlds H, Gethin $\mathrm{G}$. The psychological effects of malignant fungating wounds. EWMA Journal. 2015; 15(2):29-32.
10. Probst $S$, Arber A, Faithfull S. Coping with an exulcerated breast carcinoma: an interpretative phenomenological study. Journal of Wound Care. 2013 Jul;22(7):352-60.

11. Piggin $C$, Jones V. Malignant fungating wounds: an analysis of the lived experience. International Journal of Palliative Nursing. 2007 Aug 1;13(8):384-391.

12. Gibson $S$, Green J. Review of patients' experiences with fungating wounds and associated quality of life. Journal of Wound Care. 2013 May;22(5):265-75. 